\title{
Intra and Inter-PON ONU to ONU Virtual Private Networking using OFDMA in a Ring Topology
}

Deng, Lei; Zhao, Ying; Pang, Xiaodan; Yu, Xianbin; Liu, Deming; Tafur Monroy, Idelfonso

Published in:

2011 Asia-Pacific, MWP/APMP Microwave Photonics, 2011 International Topical Meeting on \& Microwave Photonics Conference

Link to article, DOI:

10.1109/MWP.2011.6088698

Publication date:

2011

Link back to DTU Orbit

Citation (APA):

Deng, L., Zhao, Y., Pang, X., Yu, X., Liu, D., \& Tafur Monroy, I. (2011). Intra and Inter-PON ONU to ONU Virtual Private Networking using OFDMA in a Ring Topology. In 2011 Asia-Pacific, MWP/APMP Microwave Photonics, 2011 International Topical Meeting on \& Microwave Photonics Conference (pp. 176-179). IEEE. https://doi.org/10.1109/MWP.2011.6088698

\section{General rights}

Copyright and moral rights for the publications made accessible in the public portal are retained by the authors and/or other copyright owners and it is a condition of accessing publications that users recognise and abide by the legal requirements associated with these rights.

- Users may download and print one copy of any publication from the public portal for the purpose of private study or research.

- You may not further distribute the material or use it for any profit-making activity or commercial gain

- You may freely distribute the URL identifying the publication in the public portal 


\title{
Intra and Inter-PON ONU to ONU Virtual Private Networking using OFDMA in a Ring Topology
}

\author{
Lei Deng ${ }^{1,2}$, Ying Zhao ${ }^{2,3}$, Xiaodan Pang ${ }^{2}$, Xianbin $\mathrm{Yu}^{2}$, Deming Liu ${ }^{1}$ and Idelfonso Tafur Monroy ${ }^{2}$ \\ ${ }^{1}$ Wuhan national Laboratory for Optoelectronics, School of Optoelectronics Science and Engineering, HuaZhong University of \\ Science and Technology, Wuhan, Hubei, China. \\ ${ }^{2}$ DTU Fotonik, Technical University of Denmark, Kgs. Lyngby, DK-2800, Denmark. \\ ${ }^{3}$ Department of Electronic Engineering, Tsinghua University, Beijing, China. \\ E-mail address: leide@,fotonik.dtu.dk
}

\begin{abstract}
In this paper, we propose a novel WDM-PON architecture to support efficient and bandwidth-scalable virtual private network (VPN) emulation over both inter-PON and intraPON. The virtual ring link for the VPN communications among ONUs is realized by using additionally low-cost optical passive components and OFDMA technology. Moreover, the downstream traffic wavelength is reused for the upstream traffic signal by using re-modulation technology. We report on a successful transmission of 10.7 Gbps OOK upstream and 10.7 Gbps DPSK downstream, together with 1.25 Gbps 16-QAM OFDM VPN traffic, over $20 \mathrm{~km}$ no-zero dispersion shifted fiber (NZDSF).
\end{abstract}

Keywords-Optical access networks; virtual private network (VPN); orthogonal frequency division multiple access (OFDMA); passive optical network (PON)

\section{INTRODUCTION}

The wavelength division multiplexed passive optical network (WDM-PON) technology has emerged as a future proof solution for providing broadband access [1]. In addition, it is noted that virtual private network (VPN) technology opens a door to support high speed and high quality private networking among end-users [2]. However, direct communication between optical network units (ONUs) is not supported in a traditional WDM-PON system. The inter-ONU traffic in the PON system has to be transmitted to optical line terminal (OLT) and reflected to ONUs after the scheduling and routing process in the media access control (MAC) layer. This leads increased traffic delays in the VPN, congestion at the OLT and bandwidth wastage of both upstream and downstream channels. Therefore, the realization of an efficiently physical layer VPN in WDM-PON is highly desirable to establish direct connections among ONUs [3]. Many efforts have been recently contributed to implement optical VPN communications in the WDM-PON systems [47]. One scheme reported in [4] uses the cyclic $\mathrm{N} x \mathrm{~N}$ arrayed waveguide grating (AWG) as a router for VPN and conventional non-VPN traffic, and another scheme employs frequency-tunable radio frequency (RF) tones to identify and control different VPN traffics [5]. However, these schemes can only support VPN communications within the same WDM-PON (Intra-PON). In practice, it is also very important to realize a VPN to connect ONUs in different WDM-PONs (Inter-PON). Although schemes using dynamic wavelength reflection or several fiber Bragg grating (FBG) reflectors to

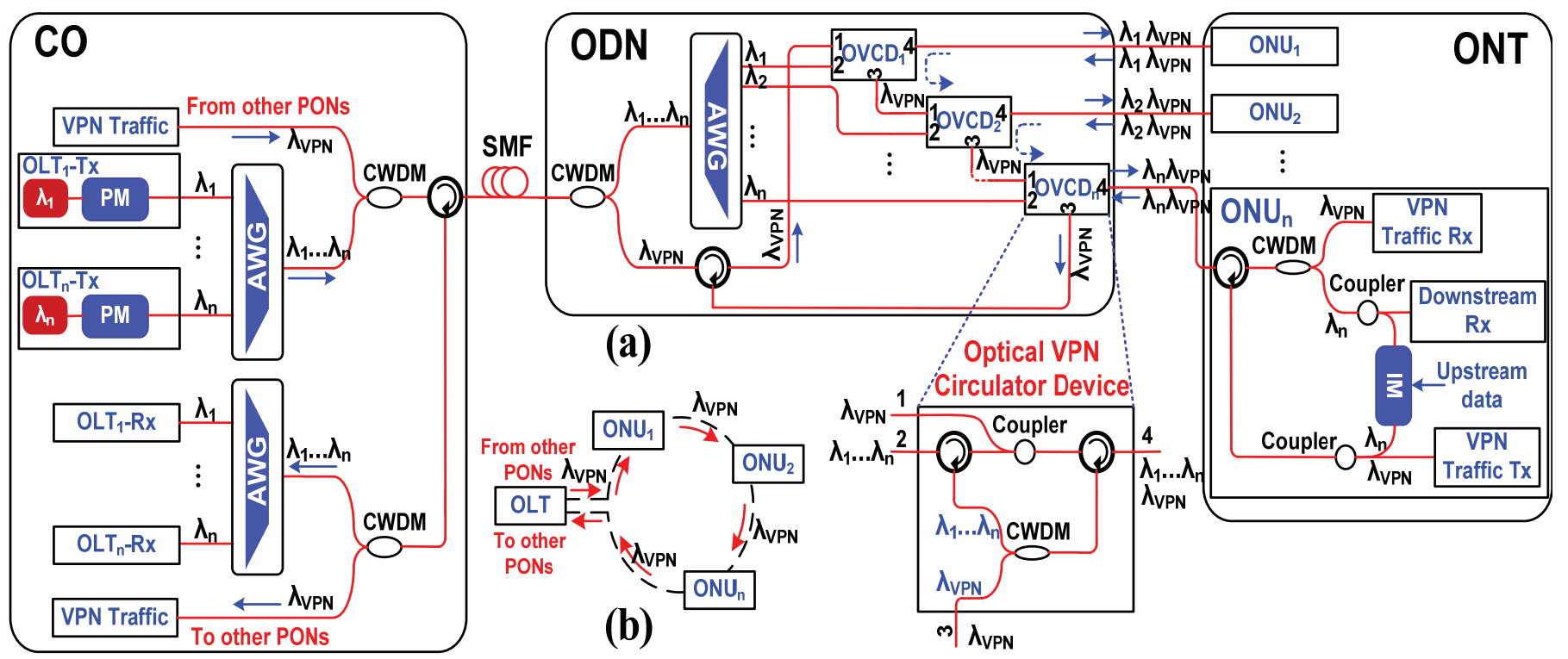

Figure 1. (a) The proposed WDM-PON architecture. (b) The virtual link for the VPN traffic transmission in the proposed system. 
establish all-optical inter-PON VPN are reported [6, 7], these two schemes architectures are impractical and very complex.

In this paper, to tackle these issues, we propose a novel WDM-PON architecture to support ring-based VPN emulation among multi-WDM-PONs. In our WDM-PON system, the onoff keying (OOK) upstream signal at an ONU is generated by reusing differential quadrature phase-shift keying (DPSK) downstream wavelengths [8] to achieve "colorless ONU" operation. By using more practical and low-cost optical passive components such as circulator, coupler and coarse wavelength division multiplexed filter (CWDM), a virtual ring link for the VPN traffic transmission among different WDMPON systems is established. Moreover, due to the use of orthogonal frequency division multiple access (OFDMA) technology for VPN traffic transmission, efficient and bandwidth-scalable VPN traffic can be supported in our scheme. Experimental results show that $10.7 \mathrm{Gbps}$ OOK upstream and $10.7 \mathrm{Gbps}$ DPSK downstream are successfully transmitted over $20 \mathrm{~km}$ no-zero dispersion shifted fiber (NZDSF) with 1.25 Gbps 16-quadrature amplitude modulation (QAM) OFDM VPN traffic for both inter-PON and intraPON.

\section{PRINCIPLE AND SyStem ARCHITECTURE}

Figure 1 (a) illustrates the proposed WDM-PON architecture. An optical distribution node (ODN) is used to connect multiple ONUs at an optical network terminal (ONT) to a central office (CO). $\mathrm{N}$ wavelengths (e.g., $\lambda_{1}-\lambda_{n}$ ) are designated for the up/downstream traffic of the $\mathrm{N}$ individual ONUs, and they are chosen to match with the transmission passbands of the 1 x N AWG. In addition, one wavelength $\lambda_{\text {VPN }}$ is allocated to convey the VPN traffic in our scheme. The optical VPN circulator device (OVCD) which is composed of two optical circulators, a $3 \mathrm{~dB}$ optical coupler and a CWDM filter at the ODN is used to combine and separate optical VPN signals $\left(\lambda_{\text {VPN }}\right)$ and up/downstream traffic in the link, meaning if an optical VPN traffic signal enters port 1 of OVCD, it is emitted from the port 4, but if optical VPN traffic signal is sent to the port 4 in opposite direction, it exits from the port 3 instead of port 1. However, optical up/downstream traffic signal $\left(\lambda_{1}-\lambda_{n}\right)$ can be bi-directionally transmitted between the port 2 and port 4 at this OVCD.

For each downstream wavelength at the OLT in the central office, the differentially preceded data is used to drive an optical phase modulator (PM) to generate the downstream DPSK signal. After single mode fiber (SMF) propagation, one part of the downstream DPSK signal is demodulated by a downstream receiver at an ONU, while the second-part of the downstream DPSK signal is re-modulated by an optical intensity modulator (IM) to generate an upstream OOK signal.

For the VPN traffic transmission, the OFDM VPN traffic from other WDM-PON systems is combined with downstream traffic by using a CWDM filter at the CO. After fiber transmission, the VPN traffic signal arrives at the $\mathrm{ONU}_{1}$ through the circulator and OVCD at the ODN. Upon having received VPN data and control information, $\mathrm{ONU}_{1}$ determines the subcarrier on which its VPN traffic data is carried, and regenerates an OFDMA signal and then sends it to the $\mathrm{ONU}_{2}$. Generally, the VPN traffic wavelength passes through all the ONUs within WDM-PON, and eventually enter other WDMPON systems as well through the ODN and OLT. Therefore, both inter-PON and intra-PON ONU communications is realized in our scheme. The virtual ring link for the VPN traffic transmission is shown in Figure 1 (b).

\section{EXPERIMENT DEMONSTRATION}

The proof-of-concept single-channel OLT-to-ONU experimental setup of the proposed WDM-PON system is displayed in Figure 2. At the $\mathrm{CO}$, a continuous-wave (CW) light at $1555.23 \mathrm{~nm}$ is fed into an optical PM, driven by 10.7 Gbps no-return-to-zero (NRZ) data with a pseudo random bit sequence (PRBS) word length of $2^{15}-1$ and $0.6 \mathrm{Vpp}$ driving voltage. Meanwhile, the baseband OFDM VPN signal for inter-PON VPN communication is generated in Matlab, and loaded from an arbitrary waveform generator (AWG) with 256 orthogonal frequency subcarriers. 16-QAM at a total bit rate of $1.25 \mathrm{Gbps}$ is employed on each subcarrier with a PRBS word length of $2^{15}-1$.After that, the baseband OFDM VPN signal from AWG is used to directly modulate a $1530 \mathrm{~nm}$ distributed feedback (DFB) laser biased at $-37.06 \mathrm{~mA}$. Subsequently, the optical downstream traffic and VPN traffic are combined and then launched into $20 \mathrm{~km}$ NZDSF after a $3 \mathrm{~dB}$ coupler and an optical circulator. NZDSF instead of SMF is used to reduce the influence of chromatic dispersion on phase-modulated signals. In addition, $1550 \mathrm{~nm}$ band wavelengths are used in the experiment for demonstration

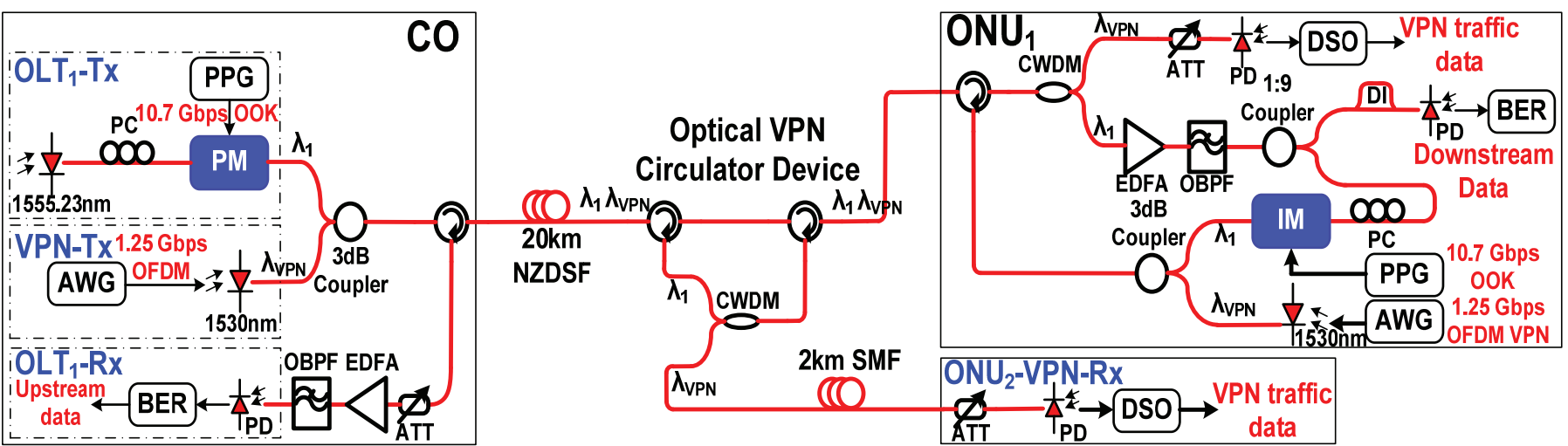

Figure 2. Configuration of the experimental setup. Pulse pattern generator (PPG), polarization controller (PC), optical tunable attenuator (ATT), optical band pass filter (OBPF), arbitrary waveform generator (AWG) and digital storage oscilloscope (DSO). 


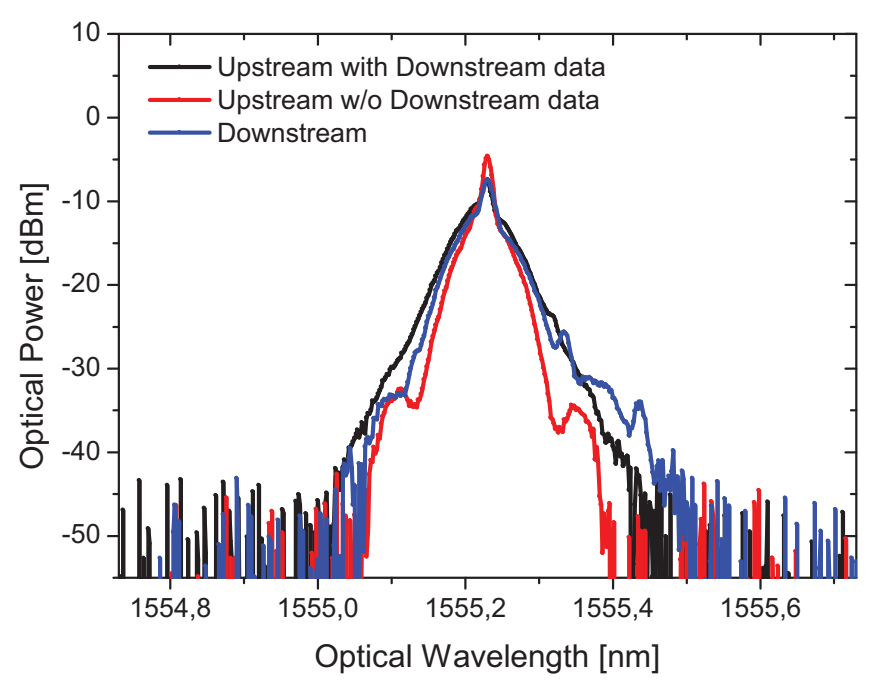

Figure 3. Optical spectra of optical downstream traffic and optical upstream traffic with or without downstream data.

purpose.

At the ONU side, a CWDM filter is used to separate the optical VPN traffic and downstream traffic. The downstream traffic signal is divided into two parts by a 1:9 optical coupler after an erbium-doped fiber amplifier (EDFA) with $15 \mathrm{dBm}$ constant-power and an optical band pass filter (OBPF) with $3 \mathrm{~dB}$ bandwidth of $0.4 \mathrm{~nm}$. For a more practical implementation, the losses of the downstream signal could be compensated by using a semiconductor optical amplifier (SOA) instead of EDFA that can be integrated into a compact module. The $10 \%$ part is input into a delay-interferometer (DI) with 93.5 ps relative delay. The demodulated downstream data from the DI is then directly detected by a $10 \mathrm{Gbps}$ photodiode (PD), and the bit error rate (BER) is estimated by a BER analyzer. In the mean time, the $90 \%$ part is fed into an optical IM, driven by a $1.6 \mathrm{Vpp}$ driving voltage and $10.7 \mathrm{Gbps}$ NRZ data with a PRBS length of $2^{15}-1$ as the upstream traffic data. The optical spectra of optical upstream traffic with or without downstream data are displayed in Figure 3, respectively.

The separated optical VPN traffic is connected to a 10 Gbps PD and sampled by a digital storage oscilloscope (DSO). Subsequently, the received VPN signal is demodulated by a digital signal processing (DSP) receiver which includes algorithms for timing synchronization, frequency and phase compensation, fast Fourier transform (FFT) and channel estimation and equalization. After $\mathrm{ONU}_{1}$ obtains its VPN traffic data, the regenerated OFDMA VPN traffic is used to modulate a $1530 \mathrm{~nm}$ DFB laser biased at $-37.06 \mathrm{~mA}$. Subsequently, the combined VPN traffic and upstream traffic are sent to the ODN, and the optical VPN traffic is separated and transmitted to $\mathrm{ONU}_{2}$ over a $2 \mathrm{~km}$ SMF by using an OVCD. Likewise, the VPN traffic signal will be demodulated at the VPN traffic receiver of $\mathrm{ONU}_{2}$. Meanwhile, the remodulated upstream traffic signal is detected by an upstream receiver, which consists of an EDFA, an OBPF, an optical attenuator (ATT) and a PD at the OLT.

\section{RESUlTS DisCUSSION}

The measured BER curves for downstream traffic with and without upstream traffic operated at $10.7 \mathrm{Gbps}$ data rate are

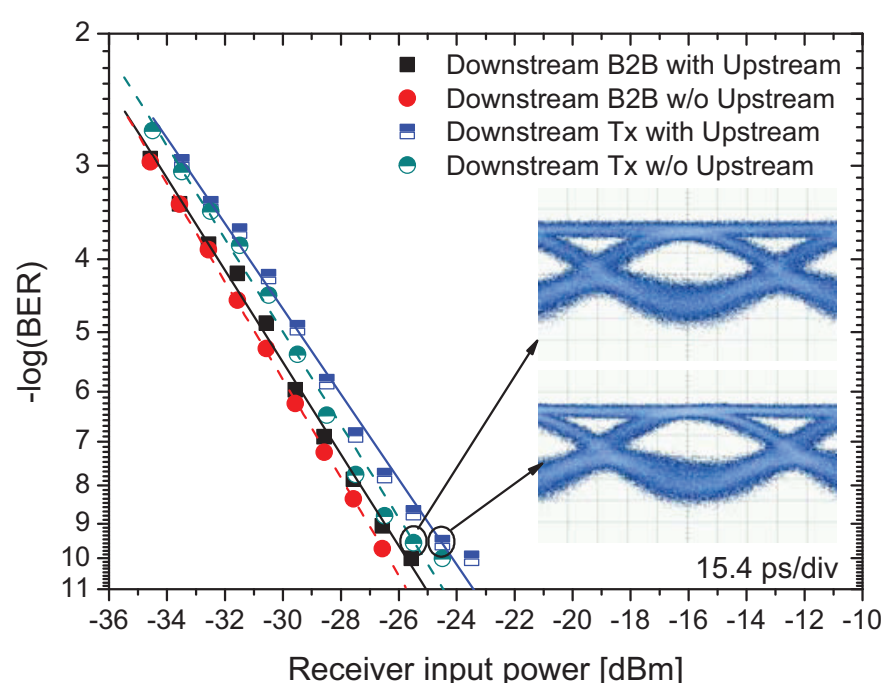

Figure 4. Measured BER curves of the downstream traffic. Tx: transmission.

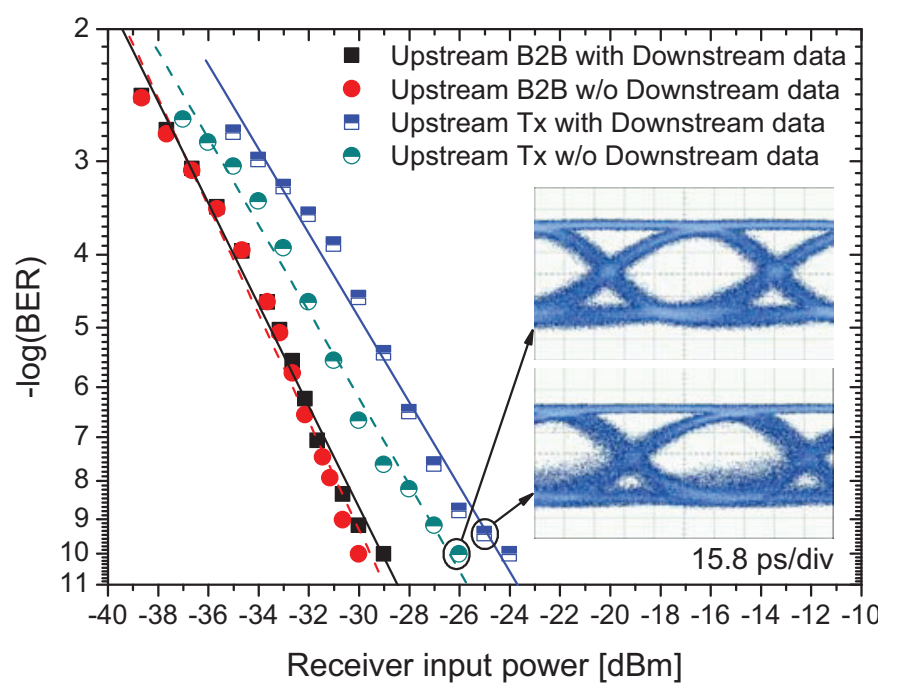

Figure 5. Measured BER curves of the upstream traffic. Tx: transmission.

plotted in Figure 4. In back-to-back (B2B) case, the measured receiver sensitivity at a BER of $10^{-9}$ is around $-26.5 \mathrm{dBm}$ with the upstream signal and $-27 \mathrm{dBm}$ without upstream signal. Copropagation of the upstream signals introduce negligible penalty of $0.5 \mathrm{~dB}$ for the $\mathrm{B} 2 \mathrm{~B}$ case. There is relatively less fiber dispersion in the link due to the use of NZDSF, therefore the downstream receiver sensitivity without upstream transmission is found to be $-26 \mathrm{dBm}$ after $20 \mathrm{~km}$ NZDSF (Tx). However, the receiver sensitivity with upstream transmission is $-25 \mathrm{dBm}$, and the $1 \mathrm{~dB}$ penalty is attributed to the optical interference noise due to the beat of the downstream signal and the Rayleigh backscattered light from the upstream signal. The eye diagrams of downstream traffic after fiber transmission are shown in Figure 4.

Figure 5 shows BER curves of the $10.7 \mathrm{Gbps}$ upstream signal. It shows that both B2B links with and without copropagation of downstream signals have almost the same receiver sensitivity of $-30 \mathrm{dBm}$, and the receiver sensitivity after transmission link without copropagation of downstream signal is $-27.5 \mathrm{dBm}$. Therefore, about $2.5 \mathrm{~dB}$ sensitivity penalty is introduced by Rayleigh backscattering in the fiber. 


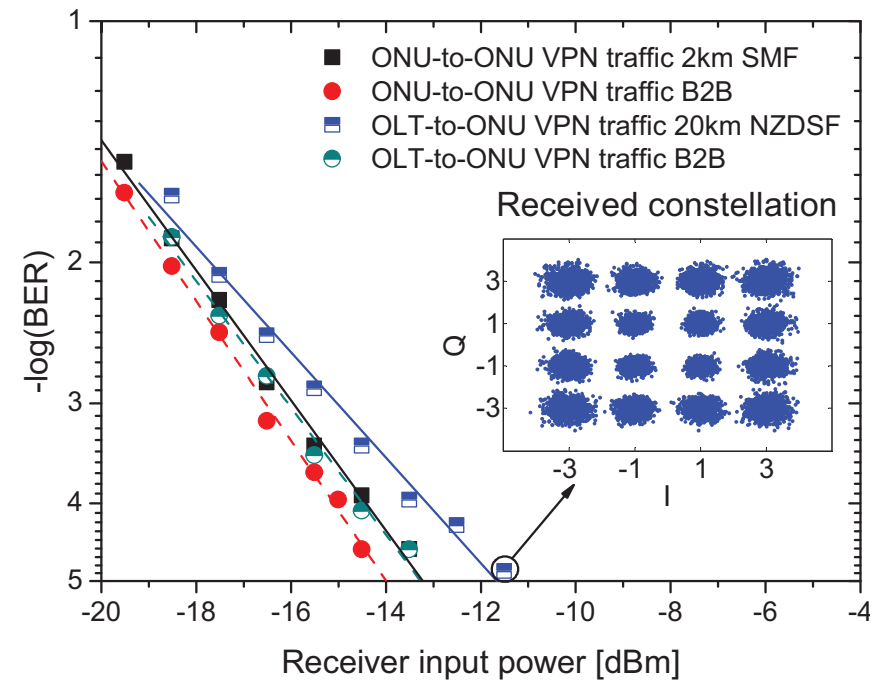

Figure 6. Measured BER curves of VPN traffic for both intra-PON and inter-PON.

Moreover, the receiver sensitivity with copropagation of downstream signal in $\mathrm{Tx}$ case is $-25.5 \mathrm{dBm}$. And the $2 \mathrm{~dB}$ penalty between transmission links with and without copropagation of downstream signals is caused by fiber dispersion that converts phase-modulated downstream signal into intensity noise at the upstream receiver. The PM-to-IM noise is clearly observed in the eye diagrams of upstream traffic in Figure 5.

We also measure the BER performance of the VPN traffic transmission for both intra-PON and inter-PON, as shown in Figure 6. It can be seen that for the B2B case, the VPN traffic receiver sensitivities at a BER of $2 \times 10^{-3}$ (forward error correction (FEC) limit) are around $-17 \mathrm{dBm}$ and $-16.5 \mathrm{dBm}$ for ONU-ONU VPN traffic and OLT-ONU VPN traffic respectively, corresponding to a negligible $0.5 \mathrm{~dB}$ sensitivity penalty. After $2 \mathrm{~km}$ SMF transmission, the ONU-to-ONU receiver sensitivity is also $-16.5 \mathrm{dBm}$. However, $-15.5 \mathrm{dBm}$ receiver sensitivity is observed for OLT-to-ONU VPN traffic over $20 \mathrm{~km}$ NZDSF. And the $1 \mathrm{~dB}$ sensitivity penalty of the OLT-to-ONU VPN traffic between B2B and transmission is caused by the fiber dispersion. The constellation diagrams of 16-QAM OFDM VPN traffic signal is shown in Figure 6 as well.

\section{CONCLUSION}

We propose and experimentally demonstrate a novel WDM-PON architecture supporting ring-based physical layer VPN emulation over both inter-PON and intra-PON. By using practical and low-cost optical VPN circulator device, a virtual ring link for the VPN transmission is established. And the application of OFDMA technology allows the efficient and bandwidth-scalable VPN traffic in our scheme. Moreover, the "colorless ONU" operation is achieved by reusing the downstream wavelength and re-modulating the phasemodulated downstream signal as the upstream signal, which could reduce the cost of physical infrastructure of our PON systems. In our experiment, error free bidirectional propagation over $20 \mathrm{~km}$ NZDSF with $10.7 \mathrm{Gbps}$ DPSK downstream traffic and $10.7 \mathrm{Gbps}$ OOK upstream traffic is successfully achieved. And the performance of upstream transmission is deteriorated by $4.5 \mathrm{~dB}$ caused by Rayleigh backscattering noise and PM-to-IM noise. Furthermore, 1.25 Gbps 16-QAM OFDM VPN traffic is also tested for both inter-PON and intra-PON cases. The proposed system provides for us a feasible and promising solution to achieve broadband VPN emulation over multi-WDM-PON systems for next generation optical access networks.

\section{ACKNOWLEDGEMENT}

We would like to acknowledge the support of the Chinese Scholarship Council (CSC) and the National "863" Program of China (No. 2009AA01A347).

\section{REFERENCES}

[1] J. Kani, M. Teshima, K. Akimoto, N. Takachio, H. Suzuki, K. Iwatsuki, and M. Ishii, "A WDM-based optical access network for wide-area gigabit access services," IEEE Commun. Mag. vol. 41, pp. S43-S48, February 2003.

[2] R. Cohen, "On the establishment of an access VPN in broadband access networks," IEEE Commun. Mag. vol. 41, pp. 156-163, February 2003.

[3] Y. Su, P. Hu, W. Hu, J. Zhang, L. Leng, H. He, X. Tian, and Y. Jin, “A packet-switched waveband-selective PON enabling optical internetworking among ONUs," Proceedings of the 31th European Conference on Optical Communication (ECOC 2009), Glasgow, U.K., September 2005, Paper We4.P95.

[4] Q. Zhao, X. Sun, Y. Ku, C. Chan, and L. Chen, "A novel internetworking scheme for WDM passive optical network based on remodulation technique," 2006 Conference on Optical Fiber Communication/National Fiber Optical Engineers Conference (OFC/NFOEC 2006), Anaheim, CA, March 2006, Paper JThB67.

[5] Q. Zhao, and C. Chan, "A wavelength-division-multiplexed passive optical network with flexible optical network unit internetworking capability,” IEEE J. Lightwave Technol. vol. 25, pp. 1970-1977, August 2007.

[6] Y. Tian, X. Tian, L. Leng, T. Ye, and Y. Su, "Optical VPN connecting ONUs in different PONs," 2007 Conference on Optical Fiber Communication/National Fiber Optical Engineers Conference (OFC/NFOEC 2007), Anaheim, CA, March 2007, Paper OWL6.

[7] Y. Tian, T. Ye, and Y. Su, "Demonstration and scalability analysis of all-optical virtual private network in multiple passive optical networks using ASK/FSK format," IEEE.Photon. Technol. Lett. vol. 19, pp. 15951597, October 2007.

[8] X. Yu, T. B. Gibbon, I. T. Monroy, "Bidirectional radio-over-fiber system with phase-modulation downlink and RF oscillator-free uplink using a reflective SOA," IEEE.Photon. Technol. Lett. vol. 20, pp. 2180 2182, December 2008. 